\title{
Outsü, enfermedades y práctica curativa ritual en los Wayuu de la Media Guajira, Colombia ${ }^{1}$
}

research paper

\section{Outsü, diseases and ritual healing practice in the Wayuu of the Media Guajira, Colombia}

\author{
Silvia Rubiela Serrano López² \\ Facultad Latinoamericana de Ciencias Sociales, FLACSO sede Ecuador \\ https://orcid.org/0000-0002-2318-0011
}

Resumen: Este artículo es producto de una investigación que tuvo como objetivo documentar en el siglo XXI las prácticas de curación indígena wayuu desarrolladas en la comunidad de Wararalain del departamento de La Guajira, Colombia. Esas prácticas las realiza la mujer outsü en contextos rituales, en medio de unas problemáticas sociales, ambientales, de seguridad y abandono por parte del Estado colombiano, en el que la cultura y la vida de todos los miembros de ese pueblo se encuentran en una constante amenaza, convirtiendo a los wayuu en personas con especial condición de vulnerabilidad. Por ser una antropóloga nativa, consideré que la metodología apropiada debía ser dialógica, por ello usé el método etnográfico que fue enriquecido con conversaciones temáticas con personas mayores, jóvenes y mujeres curadoras de una misma comunidad, en donde los espacios de diálogo los asumí como aprendizaje y a los wayuu como mis mentores. Mi argumento es que las curaciones wayuu en contextos rituales se caracterizan por la relación dialógica e intersubjetiva entre la mujer sanadora -outsü-, su espíritu auxiliar-aseyuu-, objetos de poder, el enfermo y su familia. Todo esto es posible a través de los sueños, voces y cantos como parte de la práctica curativa de su sistema de salud, ya que estos definen el tipo de enfermedad, como prevenirla y curarla. Esas prácticas curativas en la comunidad se complementan, en determinados casos, con la asistencia del enfermo al sistema biomédico que le ofrece el Estado colombiano, lo cual ha generado diálogos y conflictos entre ambos sistemas de salud. El referente teórico que utilicé como guía para la investigación involucra campos analíticos complementarios que han sido abordados desde la disciplina antropológica y las ciencias sociales, como son las discusiones acerca de lo simbólico en contextos rituales de sanación; lo onírico como categoría transversal; el chamanismo, el cual problematizo y presento las concepciones que han planteado diferentes autores y que en el caso de los wayuu esa categoría impide dar cuenta de especificidades; también analizo nociones de salud y enfermedad que algunas comunidades indígenas poseen y que se han plasmado en investigaciones etnográficas.

Palabras clave: wayuu, práctica curativa ritual, enfermedades, outsü, espíritu auxiliar, La Guajira.

Recibido: 19/11/2019| Aceptado: 28/07/2020| Disponible en línea: 15/08/2020

Como citar este artículo: Serrano-López, S. (2020). Outsü, enfermedades y práctica curativa ritual en los wayuu de la Media Guajira, Colombia. Jangwa Pana 19(2), 261 - 282. Doi:

https://doi.org/10.21676/16574923.3643

Abstract: This article is the product of an investigation that aimed to document in the 21 st century the Wayuu indigenous healing practices developed in the Wararalain community of the department of La Guajira, Colombia. These practices are carried out in ritual contexts by outsü women amid social, environmental, security and abandonment problems by the Colombian State, in which the culture and life of all the community

\footnotetext{
${ }^{1}$ Este artículo fue realizado solo con un interés investigativo en el marco de la tesis de maestría denominada "Curaciones wayuu en contextos rituales: la práctica de la outsü en La Guajira, Colombia", el cual fue favorecido con una beca de financiamiento otorgada en proceso de concurso por la FLACSO sede Ecuador.

${ }^{2}$ Correo electrónico: silviaserranosh@ gmail.com.
} 
members are in a permanent threat, turning the Wayuu people into a condition of vulnerability. Being a native anthropologist, I considered that the appropriate methodology should be dialogic. Therefore, I used the ethnographic method enriched with thematic conversations with elders, youth, and women curators from the same community, where I assumed the dialogue spaces as learning and the Wayuu as my mentors. My argument is that Wayuu healings in ritual contexts are characterized by the dialogic and intersubjective relationship between the healing woman -outsü-, her auxiliary spirit -aseyuu-, objects of power, the sick person and their family. All this is possible through dreams, voices, and songs as part of the healing practice of their health system since they define the type of disease, how to prevent and cure it. These healing practices in the community are complemented, in certain cases, with the assistance of the patient to the biomedical system offered by the Colombian State, which has generated dialogues and conflicts between both health systems. The theoretical reference used as a guide for the research involves complementary analytical fields that have been approached from the anthropological discipline and the social sciences. Among them are the discussions about the symbolic in ritual healing contexts, the dreamlike as a transversal category, and Shamanism. I problematized and presented the conceptions formulated by different authors and that, in the case of the Wayuu, this category blocks us from accounting for specificities. Finally, I analyze notions of health and illness that some indigenous communities have and that have been reflected in ethnographic research.

Keywords: Wayuu, ritual healing practice, diseases, outsü, auxiliary spirit, La Guajira.

\section{Introducción}

Según la comunidad wayuu de Wararalain que habita la parte media del departamento de La Guajira, muchas de sus enfermedades son causadas por seres espirituales del mundo-otro. Es por eso que, al sentirse enfermos por un mal espíritu, las personas deben ir ante la mujer sanadora de ese grupo indígena, la outsü, quien en el proceso ritual de sanación logra saber qué causó la enfermedad y cómo tratarla para recuperar la energía vital - $a a^{\prime}$ in - de la persona enferma. Esos conocimientos y prácticas indígenas sobre salud, enfermedad, comunicación con seres espirituales y curaciones en contextos rituales están, en gran medida, asociadas en la literatura antropológica y de las ciencias sociales a lo chamánico como noción que agrupa dichas prácticas.

Mi argumento es que las curaciones wayuu en contextos rituales se caracterizan por la relación dialógica e intersubjetiva entre la mujer sanadora -outsü-, su espíritu auxiliar aseyuu-, objetos de poder, el enfermo y su familia. Todo esto es posible a través de los sueños, sonidos, voces y cantos como técnicas curativas que caracterizan a su sistema de salud, ya que estos definen el tipo de enfermedad, cómo prevenirla y curarla. En esta práctica curativa existen objetos sagrados o de poder que configuran el contexto simbólico ritual, ya que permiten la comunicación con el mundo de los seres espirituales que indican el tipo de enfermedad y lo que el enfermo y su familia deben hacer para recuperar la energía vital y la salud del wayuu.

Ahora bien, la problemática analizada parte del conocimiento previo de la compleja realidad que vive el pueblo wayuu desde hace varias décadas. Ese pueblo está organizado en clanes matrilineales ubicados de forma dispersa en un territorio fronterizo; en una península que comprende dos países: Colombia, en el departamento de La Guajira, y Venezuela, en el Estado Zulia. Los wayuu transitan ambos países diariamente, muchos cuentan con doble nacionalidad y sus vidas se desarrollan de un lado y del otro; la división fronteriza de estos dos países no impactó su visión de territorialidad, que comprende reconocer un territorio ancestral que viene de su linaje matrilineal y el territorio que habitan por las alianzas matrimoniales, ejerciendo sus actividades culturales y familiares tanto en Colombia como en Venezuela. Son la población indígena mayoritaria en ambos países, con más de setecientos 
mil individuos entre las dos naciones (Departamento Administrativo Nacional de Estadística [DANE], 2019; Instituto Nacional de Estadística [INE], 2011).

Los wayuu en Colombia han vivido en un departamento que lucha con problemas estructurales: sociales, culturales, ambientales, políticos y durante décadas ese grupo indígena ha sido víctima de violación de derechos humanos. Entre las problemáticas que afrontan están las relacionadas con salud, marginación, violencia paramilitar, corrupción y ausencia de una política pública consistente y acorde a las realidades de quienes habitan el departamento que garantice unos mínimos constitucionales (Dejusticia, 2018; IPES Elkartea et. al., 2015).

Los miembros de la comunidad de Wararalain del pueblo wayuu de la Media Guajira aún se dedican al pastoreo de chivos y ovejos, a la siembra de algunos alimentos como la yuca, el maíz y el fríjol, pero esta actividad se ha visto afectada por la falta de lluvias en el departamento. Su sistema de organización social es matrilineal y la figura masculina que representa a la familia o al clan es el tío materno - alaula-, en el que recae la responsabilidad sobre las relaciones con otros clanes, a quienes se les lleva la palabra o el cobro de ofensas. Es él con quien se hace el acercamiento y se le presenta la intención de iniciar las alianzas matrimoniales, entre otras responsabilidades relacionadas con el desarrollo de la vida del clan. Otras de las figuras representativas dentro de la comunidad son el palabrero - pütchipüy la mujer sanadora -outsü-. El primero es el encargado de resolver las disputas entre familias. La segunda es la mujer wayuu que posee el conocimiento de curar cierto tipo de enfermedades, las provocadas por seres espirituales.

Esa comunidad, al igual que otras en La Guajira, posee saberes propios para sanar sus enfermedades que están conectados con lo onírico y el contar los sueños como característica de su cultura, debido a que en ellos los espíritus de los ancestros y otros wayuu muertos comunican hechos que sucederán a la familia, como el padecimiento de males y enfermedades (Araujo y Finol, 2010; Bilhaut, 2011; Jaramillo, 2018; Morillo y Paz, 2008; Paz, 2017; Perrin, 2011; Rincón, 2006). Las enfermedades se han complejizado en los últimos años debido a problemas sociales y ambientales: la ausencia del Estado, el consumo de agua insalubre y la escasez de lluvias en un territorio semidesértico, que han puesto en riesgo la salud de muchos wayuu. Esas problemáticas sumadas a un proyecto mineroenergético y la urbanización han reducido el territorio en el que pueden desarrollar dignamente su vida y han obligado a la comunidad a desplazarse y ubicarse en lugares cercanos a los sitios sagrados que son habitados por espíritus que causan las enfermedades.

A esto se le suma que durante décadas la Media Guajira y el departamento se ha mantenido con un alto porcentaje de pobreza extrema que para el 2017, según el DANE (2018), fue de $26,5 \%$ (el nacional 7,4 \%). Además, se evidencian las insuficientes garantías de los derechos fundamentales de toda la población que se complejizan en niños y niñas, los cuales murieron por desnutrición un total de 4770 en un periodo de ocho años, por lo cual se dictaron las medidas cautelares No. 51-15 (Comisión Interamericana de Derechos Humanos [CIDH], 2015). También se ven afectados adultos mayores y mujeres lactantes y gestantes en el acceso a salud, educación, mínimo vital del agua y alimentos, lo que convierte a los wayuu en personas con alto grado de vulnerabilidad. 
Esa realidad que muestra las dificultades en salud, el surgimiento de enfermedades y la situación medioambiental que complejiza la vida del wayuu en las comunidades dio paso a la pregunta que fue la guía de la problemática investigada: ¿Cómo los rituales, experiencias y formas de sanar de la outsü, las prácticas de sus saberes curativos y el diálogo con el sistema biomédico continúan en la vida diaria del siglo XXI en comunidades indígenas wayuu? Frente a esta pregunta, mi argumento es que la outsü habita en un mundo de saber y diálogo donde su conocimiento y práctica en rituales de sanación se define por el carácter comunitario de las relaciones intersubjetivas entre personas, espíritus y otros seres. Esto es posible a través de sueños, sonidos, voces y cantos como elementos constantes de la práctica curativa que caracterizan a su sistema de salud indígena, el cual complementan con la asistencia al sistema biomédico en determinadas ocasiones, generando así un diálogo intercultural entre estos dos sistemas.

Lo anterior me permitió dar cuenta, en la actualidad del siglo XXI, del contexto local en el que las prácticas rituales de curación indígena wayuu son desarrolladas. Dichas prácticas se mantienen activas en un departamento con múltiples problemáticas que amenazan la vida y la cultura del pueblo wayuu. Esas prácticas aún continúan vigentes a pesar de las dificultades y tensiones que ha generado la atención biomédica en miembros de la comunidad, ya que en centros de salud públicos les dan soluciones científicas a casos de enfermedades que un abuelo o abuela wayuu asociaría al robo de la energía vital por un mal espíritu, además de la forma de atención: en las clínicas esa atención es reducida a lo personal e íntimo, solo doctor/paciente, mientras que entre los wayuu su forma de sanar es de carácter comunitario y de relación con seres espirituales. Muchos wayuu en Wararalain, aún y según ciertos criterios basados en la experiencia, hacen la ponderación para decidir en qué momento ir al médico y en qué otro visitar a la outsü.

Es allí hacia donde se enfoca el objetivo de este artículo que es mostrar cómo los conocimientos sobre las formas de sanación en determinadas enfermedades y la práctica en contextos rituales de los saberes curativos de la outsü se mantienen activos en el presente siglo mediante un diálogo entre su sistema curativo y el sistema biomédico en una comunidad wayuu de la Media Guajira. Esto permitirá evidenciar, por una parte, los conflictos que se generan entre dos sistemas de salud basados en distintas ontologías; y por el otro, la manera en que una comunidad wayuu logra generar un diálogo entre esas dos formas de sanar que se expresan en la complementariedad al momento de diagnosticar y tratar determinadas enfermedades.

\section{Enfermedad, salud y conocimiento sobre curaciones rituales}

Ahora bien, la literatura correspondiente a prácticas indígenas sobre curación es amplia. En ciencias sociales existe una larga discusión etnográfica al respecto y está relacionada con estudios sobre el chamanismo, en el que se describen las formas de sanar el cuerpo, el proceso de cómo adquieren los chamanes el conocimiento y cómo conciben la salud y la enfermedad diferentes grupos étnicos (Bidou y Perrin, 1988; Eliade, 2001; Evans-Pritchard, 1976; Harner, 2016; Langdon, 1996, 2014; León, 2010; Narby y Huxley, 2005; Perrin, 1987, 1992, 2011; Sherzer, 1988; Taussig, 2002). 
En la academia ese término, chamanismo, se ha usado de forma general para referenciar espiritualidades alternativas y prácticas de sanación de muchas culturas en las que existen comunicaciones con seres del mundo-otro. Para este trabajo considero dicho término como homogeneizante, debido a que invisibiliza las particularidades de la práctica curativa ritual de las comunidades wayuu, que se basa en una interrelación entre seres humanos y no humanos que busca recuperar la energía vital, esa que habita en todos los seres vivos y cuya ausencia del cuerpo provoca enfermedad o muerte (Paz, 2007). En el caso del pueblo wayuu, la "energía vital" es aa 'in y se podría comparar con lo que en la creencia judeocristiana se conoce como alma, pero $a a^{\prime}$ ’in es más complejo y polisémico y también traduce corazón, espíritu, mente y voluntad del wayuu; es la energía vital de la persona la que sufre las enfermedades.

Con relación al pensamiento sobre estar sanos o enfermos en grupos étnicos, Bidou y Perrin (1988) han dicho que la buena salud es la coexistencia armoniosa entre el cuerpo y el alma, mientras que la enfermedad es la alteración de esta. Con más detalle, Quiroz (2015) menciona que el sistema de salud tradicional de los pueblos indígenas está definido por una visión holística que comprende cuerpo, alma, mente y espíritu, que se evidencia en los conocimientos, los procesos y las prácticas curativas y preventivas que se ejercen sobre el ser humano y en la relación de este con la naturaleza. Desde esa perspectiva, las enfermedades espirituales son vistas por la outsü como causantes de un desequilibrio en el cuerpo debido al robo del aa'in -energía vital-.

Ese tipo de enfermedades no pueden ser diagnosticadas, como tal, en la biomedicina y de proveerse un tratamiento para los síntomas, este sería ineficiente, ya que no atacaría el mal que causó la enfermedad y no habría efectividad en el procedimiento médico. Esa efectividad sí sería posible dentro de la comunidad con la atención de la curadora wayuu, a lo que LeviStrauss (1995) ha denominado eficacia simbólica. Sin embargo, existen casos en los que se acude ante los curadores propios y también al médico alijuna -no indígena, no wayuu- (Paz et al., 2010). Esos casos se presentan en el momento en que la mujer curadora de la comunidad indica que la enfermedad que posee la persona no es causada por un espíritu y por ese motivo debe ser tratada por un médico; con frecuencia esas enfermedades son infecciones, diarreas y malestares de una enfermedad común, no peligrosa.

Como los síntomas de esas enfermedades no peligrosas pueden ser similares a las provocadas por un ser espiritual, los wayuu acuden ante la outsü para que sea el espíritu auxiliar de ella quien determine qué causó la enfermedad y así poder estar seguros de ir a un médico o quedarse en la comunidad para ser atendidos por la misma outsü. Este conocimiento que posee la mujer curadora wayuu podría enmarcarse en lo que se ha denominado chamanismo, de lo cual debo decir que por mucho tiempo las investigaciones sobre este tema y sobre rituales de sanación en grupos étnicos tuvieron un sesgo androcéntrico, fueron enfocadas en gran medida en los hombres, lo que generó una invisibilización de las mujeres que también ejercían esta práctica en esas poblaciones. Este ocultamiento que tuvo la mujer en este ámbito aludió, en cierto sentido a una dificultad metodológica (investigaciones hechas por antropólogos en su mayoría hombres) y a la preeminencia de las investigaciones sobre rituales que eran dirigidos por hombres, ignorando a las mujeres chamanas y en los casos estudiados eran vistos como excepcionales, puesto que esa labor requiere cierta fortaleza que se atribuía al hombre (Colpron, 2005; Perruchon, 1997; Tedlock, 2005). 
En los wayuu las enfermedades o males ocasionados por seres espirituales pueden ser atendidos tanto por mujeres curadoras (outsü) como por hombres curadores (outshi), que son pocos; ambos tratan ese tipo de enfermedades, así como también se ocupan de hacer rituales de protección, entre otros. La investigación la orienté hacia la práctica ejercida por la outsü; sin embargo, de manera general comparto que el proceso por el que pasan los outshi como las outsü es similar: son escogidos por un ser espiritual y deben pasar por un proceso de aceptación de su espíritu auxiliar que les indica todos los procedimientos y rituales que deben hacer en cada caso.

\section{Materiales y métodos}

La metodología, por ser yo una antropóloga nativa, adoptó la propuesta de Tuhiwai-Smith (2016) sobre el redescubrir los propios saberes indígenas y también la relevancia de ese descubrimiento a la forma de llevar la vida, lo que me permitió no solo conocer las voces de los wayuu, sino también plasmar mi propia voz, mis experiencias y reflexiones. Por eso, propuse entender esta investigación como una convivencia de aprendizaje continuo en el que se "estudia con" y se "aprende de" las personas con quienes convivimos en campo, como dice Ingold (2015), sobre la antropología transformativa, en el que aprendemos a ver, escuchar y sentir cosas de la forma como los actores sociales lo hacen, proceso que transforma al investigador.

Con ese fin de ver, escuchar y sentir, planteé un trabajo de campo de tres meses en la comunidad wayuu de Wararalain en jurisdicción del municipio de Maicao, La Guajira, en la que habitan aproximadamente 16 familias. Allí también vive una outsü y la comunidad tiene constante relación con otras mujeres outsü de comunidades vecinas. Lo primero que planteé en el trabajo de campo fue hacer un recorrido en el territorio que luego se convirtió en un ejercicio constante, debido a que muchas conversaciones se daban en medio de transitar los caminos, y fue una forma de aprendizaje en campo. Así como dice Ingold (2004), el caminar es una forma de conocimiento en sí mismo, el cual puede ser leído en el territorio, porque una forma de conocer es recorrer el territorio ya que la cultura está impresa en él y no solo se conoce por medio de la mente, sino a través del cuerpo; el conocimiento se piensa, pero también se siente (Vasco, 2002).

Este trabajo tuvo en cuenta una perspectiva analítica con enfoque cualitativo, por lo cual usé como metodologías de recolección de información y análisis de datos la observación participante (Bernard, 1995), documenté rituales y experiencias de sanación llevadas a cabo por la curadora, historias contadas por miembros de la comunidad y familiares de los enfermos y conversaciones con la autoridad tradicional, docentes wayuu, familiares de la outsü y sabedoras wayuu. El criterio de selección de las personas con las que conversé fue: mujeres curadoras wayuu -outs $\ddot{u}$ - reconocidas por la comunidad; hombres y mujeres wayuu que estuvieran o hayan estado enfermos por males espirituales o no identificados como enfermedades comunes; la autoridad tradicional de la comunidad, mujeres y hombres sabedores del sistema de salud wayuu; docentes de la comunidad con conocimiento de casos tratados por la outsü; familiares, vecinos y conocidos de personas que estuvieran o hubieran estado enfermas. 
El registro se hizo por medio de notas diarias y diario de campo; grabaciones de voz y fotografías se hicieron con la previa consulta a la persona con la que se conversaba. Esta investigación priorizó las conversaciones temáticas y cotidianas, más que entrevistas semiestructuradas, debido a que los wayuu son un pueblo en el que la oralidad y el contar relatos son una forma de enseñanza que se da por medio del recordar, escuchar y conversar; en ese sentido, identifiqué durante el diseño del proyecto que formular preguntas estructuradas o semiestructuradas no iba a generar en la comunidad un ambiente familiar ni cómodo para abordar la investigación. En algunas ocasiones me apoyé en traductores de la comunidad para tener claridad de elementos específicos del ritual y el proceso curativo.

A pesar de pertenecer al clan Ipuana del pueblo wayuu del departamento de La Guajira y presentarme de esa manera, referenciando a mis antepasados ${ }^{3}$ con cada persona con la que hablaba, la investigación mantuvo, en todo momento, las normas éticas de consentimiento informado y el respeto por los modos de vida y las decisiones personales y familiares de participar o no de los miembros de la comunidad. Respeté, durante y después del trabajo de campo, "la palabra" dada y recibida, ya que los acuerdos de cualquier índole dentro de la comunidad wayuu, que hace parte de una de las características con las que se identifican, se hace por medio de la palabra, la cual es respetada y garantiza que lo dicho se lleve a cabo.

\section{Resultados}

\section{La mujer outsü en la comunidad wayuu de Wararalain}

En la actualidad del siglo XXI una de las problemáticas que se mantienen en el pueblo wayuu es el padecimiento de enfermedades comunes y espirituales que se complejiza por una débil atención en salud intercultural, debido a que muchos profesionales de la biomedicina que laboran en hospitales, clínicas e IPS indígenas y el personal del gobierno local responsable del tema de salud, poseen un conocimiento errado, parcial o nulo de las prácticas propias de sanación de los wayuu y esto impide generar un diálogo entre esas formas de sanar y la biomedicina. Fue por ello que el objetivo de la investigación se concentró en el conocimiento sobre las formas de sanación en ciertas enfermedades que hayan padecido o estuvieran padeciendo algunos miembros de la comunidad de Wararalain, así como conocer de qué manera la práctica curativa ritual que lleva a cabo la outsü y sus saberes se mantienen activos en el presente siglo mediante un diálogo entre dos sistemas diferentes de atender las enfermedades: la práctica ritual wayuu y la biomedicina.

Esa práctica curativa ritual y los conocimientos de la outsü que se desarrollan en un espacio de saberes y diálogos, tienen un carácter comunitario, el cual define las relaciones existentes entre los seres espirituales y las personas. Dichas relaciones se dan mediante experiencias oníricas y los elementos usados en el proceso curativo que definen parte del sistema curativo wayuu. En esa medida, me enfoqué en investigar los conocimientos y la práctica ritual de la mujer curadora wayuu -outsü-, quien, en muchos casos, atiende a una persona en el momento en que los análisis médicos diagnostican la inexistencia de una enfermedad, pero la persona manifiesta tener algún síntoma o cuando los síntomas no se alivian con el uso de plantas

\footnotetext{
${ }^{3}$ La manera de presentarse entre miembros de las comunidades wayuu es mencionar su clan, a sus padres, tíos y abuelos.
}

Serrano-López, S. (2020). Outsü, enfermedades y práctica curativa ritual en los wayuu de la Media Guajira, Colombia. Jangwa Pana 19(2), 261 - 282. Doi: https://doi.org/10.21676/16574923.3643 
medicinales. Ambos son un indicio de que lo que padece el wayuu es provocado por un mal espíritu.

En el momento en el que el enfermo visita a la outsü ella debe comunicarse con su espíritu auxiliar - aseyuи- para conocer la causa del mal que padece la persona y cómo recuperar su energía vital o aa'in, ya que los espíritus alteran la salud de las personas cuando intentan robar la energía vital del wayuu. La outsü se comunica con el mundo-otro, el mundo de los espíritus -pülasü-. A ese mundo pertenece su espíritu auxiliar y de ese mundo provienen los espíritus que causan muchas enfermedades e incluso la muerte de cualquier wayuu, ya sean niños, jóvenes, adultos, ancianos y, en algunos casos, hasta se encuentra en riesgo la misma outsü.

En ese sentido, la mujer curadora wayuu mantiene un diálogo constante entre dos mundos: el que vivimos -anasü- y el de los espíritus -pülasü- (Figura 1). El resultado de esa comunicación, en el que ella se presenta como un medio por el cual recuperar la energía vital perdida, es saber el tipo de espíritu que ocasionó la enfermedad, cómo y por qué la persona adquirió la enfermedad, la forma como debe proceder la familia, lo que se necesita para salvar la vida y recuperar la salud de la persona y el tipo de pago. Todo esto es comunicado a la outsü por su espíritu auxiliar-aseyuu- a través de la experiencia onírica y en medio del ritual. Lo que solicita el аsеуии de la outsü debe ser realizado de manera estricta y sobre todo el pago, que no es definido por la mujer sanadora sino por su espíritu; desobedecer trae consecuencias para la outsü, ella sería castigada por su espíritu por no hacer estrictamente lo que se le comunicó y hasta puede enfermar de gravedad.

Figura 1. Comunicación de la outsü entre los mundos anasü, jepira y pülasü

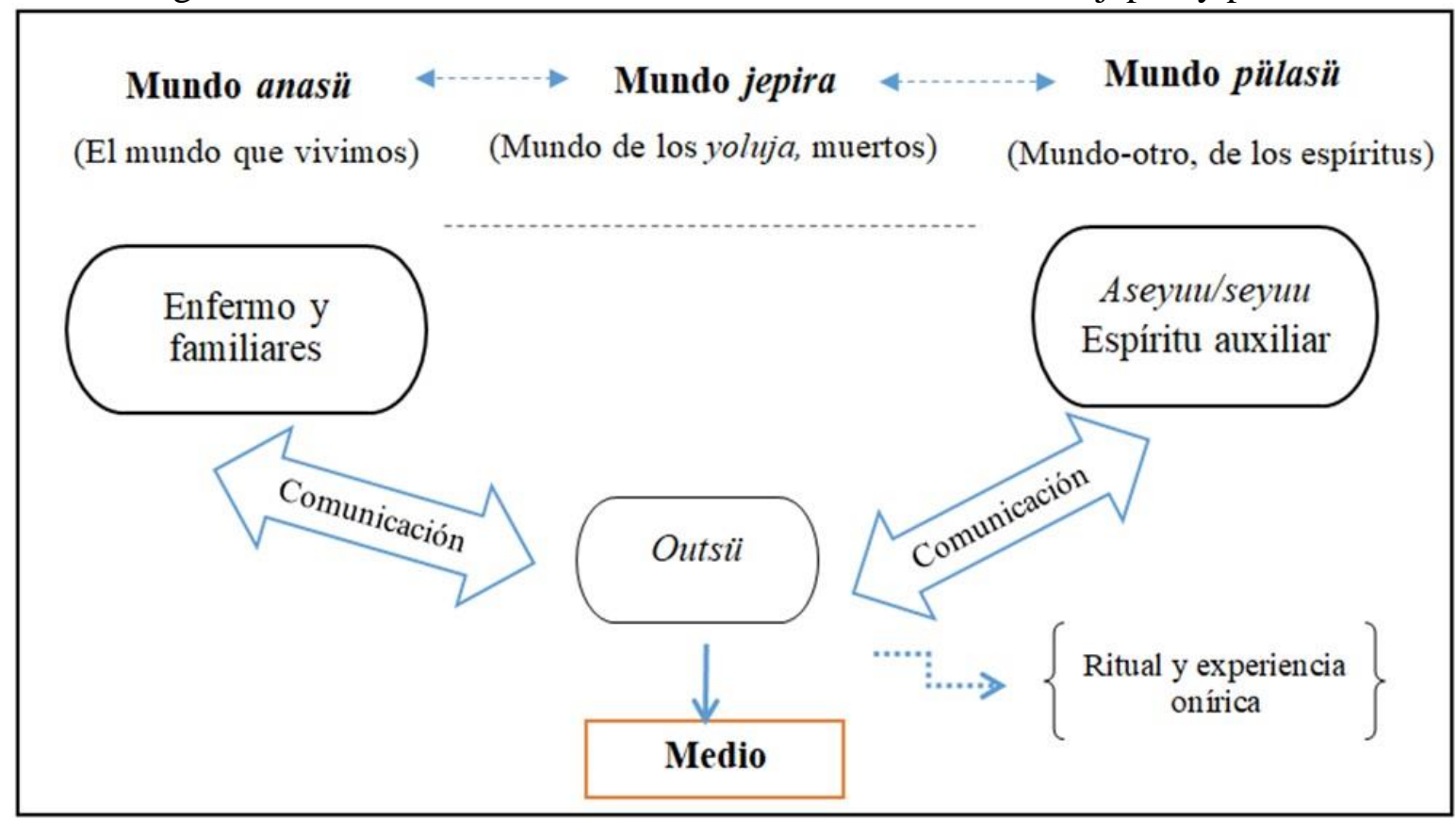

Fuente: Serrano, S. (2020). 
En la figura anterior se muestra la relación y la comunicación de la mujer outsü con dos mundos identificados por la comunidad wayuu: el mundo anasü (el que vivimos) y el mundo pülasü (el de los espíritus, el mundo-otro). El enfermo, la familia de este y la comunidad viven y se relacionan solo en el mundo anasü. Por su parte, a la outsü, quien también vive y se relaciona en el mundo anasü, le fue dado el don de comunicarse con los dos mundos por medio de su аsеyиu al cual llama en el ritual o se comunica con ese ser espiritual durante la noche, por medio de sus experiencias oníricas. El aseyuu de la outsü pertenece al mundo pülasü; es a este al que también pertenecen los seres que ocasionan los males que ponen en peligro la vida de los wayuu cuando hieren y roban su a a ’in -la energía vital-.

\section{Enfermedades que sana la outsü}

En la comunidad tenían claro que el wayuu, así como cualquier persona, podría enfermarse. Entre esas posibles enfermedades reconocían que existen unas que no son graves, que pueden atenderse en la misma casa del enfermo con algunas plantas medicinales o yendo al médico. Este tipo de enfermedades como gripes, infecciones, vómitos, resfriados, algunos dolores, etc., que pertenecen al mundo natural, a este mundo, son llamadas en la comunidad como ayulee. El otro tipo de enfermedades son causadas por los yolujas, por seres del mundo pülasü, del mundo-otro; de este tipo de enfermedades, las más conocidas en el ámbito investigativo son las wanülü̈̈ que son las más graves y malignas, pues pueden provocar la salida definitiva del $a a^{\prime}$ 'in -energía vital-del wayuu y causar la muerte. De este segundo tipo de enfermedades, que posee una clasificación con nombres más detallados para cada enfermedad, se ocupa la outsü dentro de la comunidad. El nombre con el que se reconoce cada enfermedad de tipo wanülü̈u depende del tipo de espíritu que ocasiona el mal, la forma como se adquirió la enfermedad y de la persona enferma, puesto que son diferentes las enfermedades a las que están expuestos los niños, las mujeres embarazadas, los adultos y los ancianos.

Al respecto del tipo de enfermedad que cura la outsü en las comunidades, Yaniris López me comentó que:

Con la enfermedad pülajuwa, una persona enferma a un bebé solo con mirarlo. Esta llora desesperadamente cuando quien lo mira ha matado a alguien. Como a veces no se sabe quién pasó y lo vio, es cuando buscan a la outsü y ella dice que busquen a alguien que haya matado para que lo bañe o lo sobe.

Otra enfermedad es kaliashi. Les da a los adultos. Eso puede traducir herido, eso significa que wanülü̈̈ hiere a las personas adultas y la persona se puede morir instantáneamente, otros quedan vivos, pero como si no tuvieran alma... y tienen que llevarlo enseguida donde la outsü para que recupere el alma antes de que muera. (Yaniris López, marzo de 2019, conversación)

En la conversación con Yaniris me pude percatar de que las enfermedades que atiende la outsü pueden provenir directamente del contacto con un yoluja, cuando un wanülüü hiere a un wayuu, cuando esos seres ven a los wayuu por los caminos, pero también los vivos pueden ocasionar enfermedades y son esas personas que han estado muy cerca de la muerte, que han hecho una exhumación, se han accidentado, tocado un cadáver o asesinado a alguien; es como si una persona viva transportara dentro de sí un mal que no padece ni la agobia, pero que con 
la mirada o al tocar contamina y enferma a otros, en especial a los niños, niñas y mujeres embarazadas.

Las mujeres embarazadas se identificaron en la investigación como vulnerables a enfermedades causadas directamente por un espíritu y por personas que contaminan y enferman por haber tenido contacto con la muerte. Las mujeres wayuu con las que hablé en la comunidad tenían claro que, si algún espíritu las veía o si una persona que haya exhumado los huesos de un familiar las miraba, su bebé podría sufrir. De hecho, me fueron compartidas las historias en donde a algunas mujeres de Wararalain se les adelantó el parto por esa causa. Es por ello que se cuidan de que ciertas personas las vean, pero a pesar de cuidarse, les es difícil a muchas wayuu evitar ser vistas por un yoluja, por un wanülüï. El ser vistos y heridos por seres del mundo pülasü le puede ocurrir a cualquier wayuu mientras esté de camino al molino, pastoreando sus animales, en el jagüey ${ }^{4}$, el río, las lagunas, cerca de lugares sagrados o haciendo alguna actividad en el monte.

Otra de las enfermedades causadas por seres del mundo pülasü, del mundo de los espíritus y de los muertos, que padecieron algunos wayuu de la comunidad, es la llamada mapújana ${ }^{5}$ que "es un espíritu que está debajo de la tierra". Este espíritu causa dolores que solo se alivian yendo a la outsü. Esta enfermedad la padece un miembro de la comunidad y el caso me fue compartido por Adriana Palmar:

El hermano de Esther se enfermó otra vez. Su enfermedad es del yoluja, del wanülüü, él tiene un espíritu, ese que te conté, mapújana. Él tiene mucho tiempo con eso, le da dolor en la cadera nada más, lo han llevado a donde outshii, pero no lo sanan porque él no fue cuando su enfermedad estaba reciente y ya ninguna outsü se lo puede quitar porque está duro, ya él se muere con eso, cuando el espíritu se lo quiera llevar. Esos son casos en que van tarde donde la outsü, cuando ya el espíritu está ahí y si se lo quiere llevar se lo lleva, haga lo que haga la outsü, se lo lleva. (Adriana Palmar, abril de 2019, conversación)

La conversación con Adriana me reveló la atención temprana que deben tener las enfermedades causadas por los seres del mundo pülasü, y en ese sentido, el visitar a la outsü a tiempo para recuperar la energía vital del wayuu, ya que si el espíritu logra entrar al cuerpo de la persona y apoderarse de su aa'in, probablemente el espíritu auxiliar de la mujer curadora wayuu no podría recuperarla y el tiempo de vida del enfermo dependería del espíritu, de cuando este decidiera terminar con la vida de la persona.

Una de las outsü a la que visité contó que muchos wayuu, hombres y mujeres, la buscaban porque tenían una enfermedad llamada mmárülas; la outsü dijo que esa enfermedad era grave, tanto así que el wayuu está en riesgo de muerte. En esos casos, para curar a las personas y recuperar su aa 'in -energía vital-, el espíritu auxiliar-aseyuu- le dijo a la curadora wayuu que la única forma de evitar que la persona muriera era cambiándole el nombre. Adriana Palmar, traduciendo lo que decía la outsü, dijo:

\footnotetext{
${ }^{4}$ Reservorios de aguas lluvias.

${ }^{5}$ Sobre este ser espiritual y enfermedad, hasta el momento no existen registros en la etnografía ni en la literatura antropológica wayuu. 
Exactamente, es como si lo bautizara. Enolia no se llamaba Enolia, sino Rena, entonces el espíritu de ella se llama Enolia y le puso el nombre del espíritu a la paciente para que el mismo diablo no la vea como la había visto, para que no la reconozca, y Minko igual, es el nombre del espíritu, Gladis, Blanca, Beatriz igual, imagínate un poco que hizo ella. El de Gladis es Lalia pero le dicen Gladis. Ella le dice al enfermo: tu nombre va a ser este si te quieres sanar, ese es el nombre que tú tienes que aceptar y con ese nombre es que te van a llamar, no con el nombre antiguo, si te llaman con el nombre antiguo ya tú verás. Entonces, ¿qué prefieres tú, la vida o la muerte?, la vida, entonces tienes que procurar que te llamen con el nombre que te digo. (Adriana Palmar, febrero de 2019, en conversación con Ísana)

En el caso anterior, la outsü me compartió una de las formas como evitó enfermedades peligrosas que provocarían la muerte. Esa enfermedad denominada mmárülas la padecieron varias personas en la comunidad y el espíritu auxiliar de la outsü en cada caso le indicó que debía cambiarles el nombre a esas personas. El nuevo nombre era el mismo del aseyuи espíritu auxiliar- que intervenía en el proceso de curación; esa curadora wayuu llegó a tener más de diez espíritus.

\section{La práctica curativa ritual wayuu}

En casa de un wayuu el padecimiento de una enfermedad wanülü̈u se reconoce por los síntomas que se perciben en el cuerpo de la persona, los cuales son constantes y no se alivian con plantas ni medicinas recetadas por un médico. Aun así, en muchos casos, esos síntomas no son reconocidos de inmediato, pero visitar a la outsü determina lo que realmente tiene el enfermo. La curadora wayuu siempre busca conocer lo que causó los síntomas, más específicamente, qué tipo de espíritu, yoluja o wanülüü está tratando de robar la energía vital del wayuu.

Esa respuesta sobre lo que provocó la enfermedad a la persona se da luego de la comunicación que entabla la outsü con el mundo-otro, el mundo pülasü, ya sea por medio de la experiencia onírica o masticando tabaco, usando chirrinchi ${ }^{6}$, agitando la maraca-ishira-, y pronunciando unos cantos y palabras ininteligibles para llamar a su espíritu auxiliar o aseyuu, que son elementos simbólicos (Turner, 1999) que permiten llevar a cabo el ritual. En ese proceso se genera un diálogo entre ella y su espíritu, quien es el que le comunica la causa de los síntomas y detalles de la enfermedad de la persona, ya que, en muchos casos, los síntomas son similares en varias enfermedades, pero es el aseyuи de la outsü el que sabe exactamente qué tipo de mal padece el wayuu y qué tipo de espíritu intenta apoderarse de su energía vital.

Es por ello que, frente a cualquier síntoma persistente, lo primero que debe hacer el wayuu es visitar a la outsü. Ella en ese momento da paso al proceso ritual de sanación que inicia con la llegada del enfermo y un familiar ante la outsü o solo del familiar, en caso de que la persona enferma no pueda ir. Ella a solas se comunica con su aseyuu; según Yoni, "Isana tiene su canto con la maraca, uno la escucha, pero no la ve" ". Luego de comunicarse con su espíritu auxiliar, la outsü pide que el enfermo entre al lugar donde ella se encuentra y allí dentro

\footnotetext{
${ }^{6}$ Bebida alcohólica artesanal, producto de la fermentación y destilación de la panela que es hecha a base de la caña de azúcar.

${ }^{7}$ En conversación con Yoni, marzo de 2019.
}

Serrano-López, S. (2020). Outsü, enfermedades y práctica curativa ritual en los wayuu de la Media Guajira, Colombia. Jangwa Pana 19(2), 261 - 282. Doi: https://doi.org/10.21676/16574923.3643 
informa todo lo que le ha dicho su aseyuu. El enfermo regresa a su casa para conseguir lo que se le ha pedido y debe volver para ser bañado y continuar el proceso de sanación. En otros casos, los familiares, tías, madre o abuela, del enfermo le hacen el proceso de sanación en su casa, tal como lo ha indicado el espíritu auxiliar de la outsü; lo bañan, lo encierran por varios días, si es el caso, y le preparan los alimentos de la dieta, que en muchos casos debe hacer la persona para recuperarse. Con respecto a la forma de curar, Yoni me contó que:

Para curar es una conversación de ella con el espíritu y en ese dialogo el espíritu pide un chivo de algún color y da el color que quiere, lo que importa es que se derrame la sangre de ese animal que pide. Es ahí mismo que se nota la efectividad que indica que te va a curar, si no tú sigues lo mismo, no cambias de estado. (Yoni, marzo de 2019, conversación)

Lo que comentó Yoni hace parte de lo que puede indicar el espíritu auxiliar de la outsü en el proceso de sanación. Ese ser espiritual puede pedir el sacrificio de chivos de colores específicos, puede pedir que se realice la danza ritual wayuu o yonna, pedir que se consigan ciertos elementos como collares, telas rojas, etc. Esas indicaciones son vistas por el enfermo y su familia como un deber, una obligación y, al mismo tiempo, una necesidad, por dos razones: uno, el cumplir estrictamente con lo que indica el espíritu auxiliar de la outsü garantiza recuperar el $a a^{\prime}$ 'in o energía vital y así sanar definitivamente. Dos, desobedecer esa indicación pone en riesgo la salud y la vida de la curadora wayuu, puesto que su espíritu auxiliar la castiga, la hiere, la enferma de gravedad porque lo demandado por él no se hizo como lo comunicó.

Como aparece en párrafos anteriores, la experiencia onírica es una forma en la que se manifiestan los espíritus de los wayuu que han fallecido (yoluja) y comunican lo que va a suceder, la forma de prevenir o tratar una enfermedad o una situación, y les muestran a algunas personas las plantas y sus beneficios. Estas experiencias las pueden tener las mujeres y hombres curadores, las personas con especialidades de sanación que mencionaré más adelante, miembros de la familia del enfermo, el enfermo mismo y personas de la comunidad. El sueño, más que una experiencia que se da en altas horas de la noche es una deidad. Al respecto de los sueños y los procesos de curación, Paz (2017) menciona que en los wayuu es por medio de Lapü (deidad del sueño) que "se pronostican muchos de los sucesos que acontecerán sobre salud, enfermedad, vida, muerte, de ahí que esta deidad sea fundamental para los oütshii, piaches o curadores wayuu" (p. 278).

Con referencia a la interpretación de los sueños, existen algunas mujeres en las comunidades que son reconocidas por ser sensibles al lenguaje onírico y saber con exactitud lo que dicen los sueños. Sin embargo, lo que con frecuencia dicen las mujeres y los hombres wayuu es que los sueños son claros y en muy pocas ocasiones necesitan interpretación. En los sueños te muestran, te previenen y te dicen lo que debes hacer. Los sueños son una guía de las actividades diarias del pueblo wayuu, tanto así que el primer saludo por la mañana, al levantarse, la abuela o la madre pregunta: jamaya pü 'lapüin-¿qué tal estuvo el sueño?- 


\section{La práctica curativa ritual wayuu y la biomedicina}

El universo del sistema curativo wayuu es amplio y en él se encuentran varios curadores o especialistas, diferentes a las o los outsü (shi). Según investigaciones hechas por Paz (2007), hay una subdivisión de especialidades dentro de ese sistema de salud y menciona los siguientes:

Emeijut (la especialista en partos), Epitutu o'uupala (curación de órganos como los ojos), Oulakut (la que ejerce la adivinación), Anaajüt jipu (especialista en huesos), A'latülü (masajeadora), Jupula tepichi (especialista en niños), Anaajülü ale'e (la partera), Alajut ипи'u (especialista en medicina Wayuu). (p. 132)

A las anteriores especialidades se le suma la outsü o el outshi que son los guías espirituales en la vida de los wayuu dentro de las comunidades. Se encargan de diagnosticar, dar tratamiento y curar males causados por seres del mundo pülasü, pero también de hacer rituales de protección. Aunque existen hombres y mujeres wayuu que fueron escogidos por un азеуии para dedicarse a guiar la vida espiritual de ese pueblo, las comunidades ven a las mujeres como las encargadas de la protección y del cuidado de las familias y la comunidad en general. Esto se refleja en los diferentes rituales de la vida del wayuu en el que es la abuela, la tía o la madre las que, según sea el caso o lo dicho en sueños, se encargan de llevarlos a cabo.

En la comunidad de Wararalain las formas de sanación más utilizadas son la práctica curativa propia y la biomedicina. En el momento en que aparecen los síntomas de una enfermedad, que suelen ser similares los que causa un espíritu con una enfermedad no grave, la mayoría acude primero ante la outsü y es ella la que determina si la enfermedad fue causada por un ser del mundo pülasü o si es una enfermedad que debe atender un médico. La razón de esa decisión se fundamenta en el hecho de evitar un posible fallecimiento en un centro de salud, ya que, si es un espíritu el que se encuentra causando los males al cuerpo del enfermo, la vida de esta persona correría peligro, debido a que este será inyectado para extraerle sangre o suministrarle un medicamento y ese cuerpo extraño, la aguja, es un elemento que rechazan los espíritus causantes de las enfermedades. Esa acción asegura la muerte del paciente y es a lo que le temen los wayuu.

A pesar de ese riesgo conocido por todos en la comunidad, hay quienes acuden primero al sistema biomédico, que es gratuito por ser indígenas, y lo hacen por falta de recursos económicos o por falta de animales que le permitan asumir los costos de la cura que pide el espíritu auxiliar de la outsü y en muchas ocasiones deben activar las redes de solidaridad familiar para cubrir los gastos tanto en los requerimientos de la mujer curadora wayuu como los que se generan en el pueblo al momento de asistir a una clínica u hospital.

Durante el trabajo de campo encontré casos de padecimientos de más de una enfermedad y situaciones que debían ser atendidas tanto por la outsü como por un médico. Ese proceso me reveló una forma de articulación o complementariedad entre la práctica curativa ritual y la biomedicina. En el momento en que se presentan esos casos de una enfermedad que puede ser tratada por ambos sistemas de salud (cultural y biomédico), el enfermo visita a la outsü para que ella recupere su energía vital y el espíritu salga de su cuerpo, luego la misma 
curadora le indica a la persona que se encuentra fuera de peligro, que asista al médico para que cure la enfermedad que a este le compete, que en muchas ocasiones son infecciones o malestares físicos.

Pude identificar que la complementariedad entre esos dos sistemas de salud es asumida desde la comunidad y su curadora wayuu hacia la atención biomédica, ya que reconocen que ciertas enfermedades no son competencia de la outsü y ella no las puede sanar. Es por ello que asisten a las clínicas y hospitales, siempre que estén seguros de que no están en riesgo de que un espíritu les cause la muerte por ser inyectados. En cambio, desde la biomedicina hacia la práctica curativa propia del wayuu es inexistente una articulación o un diálogo intercultural real, debido a las bases científicas de la disciplina médica y a los procedimientos de atención establecidos que desconocen las concepciones wayuu de familia, comunidad, salud, enfermedad y las relaciones que estas tienen con los sueños. En ese sentido, se generan conflictos entre los dos sistemas de salud, ya que, para muchos médicos no wayuu, las outsü asocian una enfermedad que tienen diagnósticos y tratamientos específicos en la biomedicina con enfermedades provocadas por espíritus.

Lo anterior muestra la existencia de dos formas distintas de alcanzar un bienestar para uno corporal/espiritual y para el otro solo físico. Cada uno de esos sistemas de salud se desarrolla en contextos culturales distintos que están inmersos en relaciones de poder y dominación; en este caso la que ejerce la biomedicina como parte de la cultura mayoritaria, la no indígena, sobre las formas de curación wayuu. Esa relación de dominación dificulta la consolidación de un diálogo intercultural, ya que ambos sistemas de salud tienen sus bases en diferentes epistemologías, y hay que tener en cuenta que quienes ejercen la actividad de sanación en los wayuu no son análogos al médico, así como las plantas no son análogas a los fármacos, son diferentes.

Ahora, el contexto social, político y ambiental al que se encuentran expuestos los wayuu de la Media Guajira desde hace muchos años y que los han colocado en situación de vulnerabilidad, se caracteriza por la dificultad de acceder a alimentos que les proveía la tierra, la falta del recurso hídrico por la ausencia de las lluvias y las sequías extremas, impactos en el uso del territorio por la explotación de recursos naturales a cargo de empresas privadas, y otros aspectos que desencadenaron múltiples situaciones entre las que se encuentran las problemáticas en la salud, el recurrente padecimiento de enfermedades tanto ayulee como wanülüü y enfermedades nuevas, para las cuales no tienen una cura o tratamiento en las comunidades.

En el caso de la comunidad de Wararalain, el $99 \%$ de las personas, en el momento de la investigación, se encontraban afiliadas a las Instituciones Prestadoras de Servicios de Salud Indígena (IPSI) ubicadas en el municipio de Maicao. Algunas de las clínicas tienen como visión prestar el servicio con enfoque diferencial y con el objetivo de integrar los saberes curativos wayuu con la biomedicina. Según los testimonios de la autoridad tradicional de la comunidad y algunos de los habitantes de Wararalain, las atenciones médicas en estas IPSI son prioritarias para los y las wayuu, las citas médicas se las programan para el mismo día que las solicitan de manera presencial, debido al hecho de que muchas de estas personas se trasladan desde sus comunidades con dificultades y no cuentan con recursos para movilizarse de manera constante. 
La IPSI mantiene en seguimiento y control a las mujeres en estado de gestación y en muchos casos las van a buscar a sus comunidades para que cumplan con sus controles y las del recién nacido. También cuentan con un profesional que de manera general les transmite información en su idioma, pero dentro de los consultorios deben ingresar con familiares que sepan hablar español, para el caso de los adultos mayores que solo hablan wayuunaiki.

Los miembros de la comunidad de Wararalain al respecto de las atenciones médicas recibidas por la IPSI evidenciaron lo siguiente:

Cada tres meses llega una brigada de salud de Anas Wayuu para atender a todos los miembros de la comunidad desde las ocho de la mañana hasta las dos de la tarde, con los servicios de odontología, citología, médico general y farmacia.

El servicio de citología ya poco viene porque a las mujeres no les gusta hacérsela. (Testimonio de la comunidad de Wararalain, marzo de 2019)

Al preguntar el por qué las mujeres de esa comunidad no usaban el servicio de citología que llegaba con las brigadas de salud, me contaron que el profesional médico que los visitaba para hacerles la citología era un hombre y que culturalmente no está bien visto que la mujer esté desnuda delante de un hombre y menos aún si esa mujer ya está casada.

Ahora bien, el sistema biomédico en el departamento de La Guajira ha buscado implementar articulaciones entre la salud indígena wayuu y la biomedicina, que se ha enfocado en facilitar el acceso a la salud teniendo en cuenta las características culturales de ocupación del territorio que se da de forma dispersa; respetan las decisiones y la libertad que tiene cada wayuu para escoger la forma de recuperar su salud. Aunque existen casos en los que los médicos consideran que deben intervenir y convencer a las familias wayuu de que la mejor opción es la biomedicina, estos casos son a menudo los relacionados con la desnutrición de niños y niñas, ya que el personal médico ingresa a las comunidades y deben salir con los pacientes que requieren hospitalización con urgencia. Esto genera conflictos en las familias de los menores que temen que sus hijos e hijas tengan una enfermedad provocada por un ser espiritual y que al ser llevados a un centro médico mueran de inmediato.

\section{Discusión}

Las evidencias de la investigación me permitieron analizar las formas en las que los miembros de la comunidad wayuu de Wararalain en el presente siglo XXI mantienen activa la práctica curativa ritual en la que las relaciones intersubjetivas, que se dan en medio del diálogo entre personas y seres del mundo-otro, poseen un carácter comunitario que se activa desde el inicio del proceso curativo que lleva a cabo la outsü. Esa práctica ritual de sanación encuentra unos momentos de articulación y de conflictos o tensiones con el sistema biomédico en situaciones en que las enfermedades que padece un wayuu deben ser tratadas en los dos sistemas de sanación: el propio y el de occidente. Estudios realizados en Venezuela sobre el pueblo wayuu resaltan que el sistema biomédico es otra de las opciones que tienen miembros de estas comunidades para tratar sus enfermedades (Balza-García, 2010; Paz, 2007; Paz et al., 2010; Rincón, 2006). Esos estudios también desarrollan un análisis acerca de la confianza del wayuu en los centros de salud y lo ineficaz que resulta ser la biomedicina 
en algunos casos donde la única opción de cura la tiene la outsü por medio de su espíritu auxiliar.

En la práctica curativa ritual wayuu la outsü posee el don de comunicarse con el mundo pülasü, para saber qué enfermedad tiene la persona y cómo tratarla. Las enfermedades en este caso son entendidas como una energía negativa o un espíritu que ingresa al cuerpo de una persona con la intención de extraer la energía vital (Ojeda, 2013). Según los wayuu, este tipo de enfermedades no pueden ser diagnosticadas como tales desde la biomedicina, porque en ellas no tienen competencia los médicos y el tratamiento que proporcionen no sería eficaz para recuperar la salud perdida. Esa efectividad en el diagnóstico y en el tratamiento que logra la outsü es denominada por Levi-Strauss (1995) como eficacia simbólica, donde la persona enferma y la comunidad en la que vive creen en un sistema relacionado con seres sobrenaturales que funda la concepción indígena del universo, donde la enfermedad, los dolores y las afectaciones a la salud son elementos extraños que un chamán interviene para alcanzar la cura.

La noción de chamán en la literatura antropológica es utilizada por autores como Bidou y Perrin (1988), Levi-Strauss (1995), Narby y Huxley (2005), Taussig (2002), entre otros, para referirse a seres humanos con poderes sobrenaturales que por medio de actos mágicos curan a las personas (Sherzer, 1988). Al respecto, Almendro (2008) menciona que cualquiera que sea la definición que se desarrolle sobre el chamán, esta hará referencia a poderes especiales, a la ayuda de espíritus, a la capacidad de contacto con el mundo-otro y hasta de hacer su aporte como guía. Estas nociones, hasta cierto punto, son problemáticas al colocarlas en diálogo con la práctica curativa ritual wayuu donde la actividad de la outsü es más compleja, puesto que está relacionada con concepciones de enfermedad, salud, vida y muerte que hacen parte de su propia ontología como pueblo indígena, que supera los actos mágicos que menciona Sherzer (1988), ya que la eficacia de la sanación en la comunidad wayuu se debe a la sabiduría de la outsü a quien le fue dado un don desde el momento que aceptó el espíritu auxiliar que la acompañaría hasta su muerte y con quien se mantiene en comunicación y en proceso de aprendizaje continuo. En ese sentido, no hay cabida para la adivinación ni el azar; todo el proceso de diagnóstico, curación y pago se hace porque un espíritu lo ha indicado y enseñado a la outsü con el fin de recuperar la energía vital del enfermo.

Sobre la eficacia en la sanación wayuu, Perrin (1988) identificó tres componentes: la de orden intelectual y religioso, que recoge la concepción wayuu de la enfermedad y las relaciones entre el mundo de los espíritus y el nuestro; la de orden económico y social, las formas de pago y las redes que se activan alrededor de las necesidades del enfermo y su familia; la del cuerpo y las sensaciones. Esos tres componentes, según el autor, definen la eficacia terapéutica de la práctica curativa ritual wayuu. La interpretación que hace Perrin (1988) asume dos posturas que se interconectan: una es la intención de la curadora wayuu de sanar a la persona siguiendo las indicaciones de su espíritu auxiliar. La otra, es la confianza del enfermo y su familia en que la outsü recuperará su energía vital o aa ’in.

Para el wayuu, estar enfermos a causa de un wanülüü o cualquier otro ser del mundo pülasü significa que su energía vital o aa 'in está siendo perturbada y extraída de su cuerpo. La aa 'in está relacionada con la vida, la muerte y la enfermedad; su separación del cuerpo produce la enfermedad y en otros casos la muerte (Paz, 2017). Es por ello que la outsü lo primero que 
trata de establecer es qué espíritu ocasionó el mal y qué caminos transitó la persona en donde pudiera ser visto por algún yoluja (Castro, 2017), para saber si es posible recuperar la energía vital y de qué manera hacerlo. Todo para que el wayuu vuelva a estar sano, y eso solo lo logra la curadora wayuu en el momento en el que la energía vital del enfermo es recuperada en el proceso ritual.

Ese proceso curativo ritual en el que la aa'in es recuperada se produce en medio de relaciones, acciones y objetos simbólicos que, como dice Turner (1999), conservan las propiedades del ritual y que puestos en diálogo configuran la práctica de sanación wayuu. Todos esos elementos del contexto simbólico permiten la comunicación con el mundo-otro, el mundo de los seres espirituales que indican el tipo de enfermedad y lo que se debe hacer para que la persona recupere su energía vital y su salud. Esos elementos, personas y seres que intervienen en el ritual y el proceso de sanación se presentan en medio de un diálogo y de relaciones intersubjetivas que continúan durante más tiempo que el empleado para llevar a cabo el ritual, ya que el espíritu de la curadora recupera la energía vital de la persona enferma, le devuelve su salud, pero a cambio se debe hacer o entregar lo que el espíritu auxiliar exija, como sacrificios de animales, entrega de joyas, hilos y tela roja, entre otros; también, en algunos casos, se deben hacer baños en determinadas horas. Lo dialógico se presenta en el acto comunicativo de la curadora con su espíritu auxiliar y con el familiar que acompaña a la persona enferma y con el mismo enfermo, si este no es un menor. Es un diálogo que hace parte del diagnosticar qué causó la enfermedad.

La acción comunicativa entre los que intervienen en la curación se da en dos espacios íntimos que son compartidos a través de la oralidad: uno es el que se presenta en el mismo ritual, donde la curadora wayuu a solas usa los objetos simbólicos como la maraca, el tabaco, el chirrinchi y el cintillo; y el otro, reflexionado desde las propuestas teóricas y conceptuales de Bilhaut (2011), Paz (2017) y Perrin (2011), se presenta por la noche, en la experiencia onírica, donde la outsü, el enfermo y un familiar de este tienen la posibilidad de soñar y ser contactados por seres del mundo-otro, del mundo pülasü, del cual les transmiten mensajes relacionados con la enfermedad y padecimientos del wayuu.

Las funciones de los objetos en el ritual en el primer espacio son generar sonidos con la maraca que permiten llamar a los aseyuu; develar el sabor del tabaco como planta sagrada y del chirrinchi, así como los olores de estos, que son sensibles a los espíritus y con ellos la curadora logra conectarse con el mundo pülasü. El cintillo, que es de color rojo con cuentas que cuelgan, actúa como elemento protector de la outsü. Todos esos elementos son usados para activar el diálogo con su аseyuи o espíritu auxiliar.

Los espacios de comunicación mencionados (la conexión íntima de la outsü con su espíritu auxiliar y la experiencia onírica como una forma de revelación de lo que acontecerá con una familia), tanto en problemas de salud como claniles, son espacios que asumen una conexión espiritual con un mundo-otro y es este aspecto de la práctica curativa ritual wayuu la que genera tensión en profesionales de la biomedicina en momentos en que para el wayuu la articulación entre su sistema curativo propio y el del alijuna -no indígena- es necesaria.

En esta investigación identifiqué la compleja situación que genera en el wayuu enfermo y su familia no ser comprendidos en el momento en que deciden no acudir al médico por temor a 
que el enfermo sea inyectado o intervenido su cuerpo y en esa medida acelerar la muerte de su familiar. En esas situaciones solo acuden a la curadora wayuu de su comunidad. Aunque existen casos en los que el espíritu auxiliar de la outsü no ve la enfermedad de la persona, esto obliga a los familiares del enfermo a buscar referencias de una outsü en otras comunidades que pueda decir qué tipo de enfermedad tiene la persona. En esa búsqueda pueden conseguir a un outshi, un hombre que cura esas enfermedades. Entre los wayuu de Wararalain tanto mujeres como hombres curadores - outshii-son reconocidos con igual nivel de sabiduría y don para atender las enfermedades provocadas por seres del mundo pülasü, pero, según miembros de la comunidad, son más las mujeres que los hombres quienes tienen ese don, que son escogidos y aceptan al espíritu. En esta investigación atendí a esa premisa y lo identifiqué como un aporte a la literatura antropológica y de las ciencias sociales sobre los conocimientos de la mujer en la práctica curativa, debido a que las investigaciones de este tipo tuvieron por mucho tiempo un sesgo androcéntrico, fueron enfocadas en los hombres chamanes y de esa manera estuvieron invisibilizadas las mujeres que también ejercían estas prácticas en las poblaciones.

Ese ocultamiento, en cierto sentido, se produjo por una dificultad metodológica al ser los antropólogos en su mayoría hombres y por el predominio de investigaciones que se concentraron en las prácticas rituales dirigidas por hombres. A pesar de ello, existen investigaciones (Colpron, 2005; Perruchon, 1997; Tedlock, 2005) que cuestionan con datos empíricos la visión androcéntrica en los trabajos sobre el chamanismo y que además revelan que la mujer ha estado por décadas en los espacios rituales como actor activo e influyente en sus comunidades. Otras investigaciones muestran que mujeres y hombres son un complemento en las curaciones, hacen parte de un trabajo en conjunto en el que no se define dominación de poderes o de conocimientos de uno sobre el otro.

En el caso de la comunidad de Wararalain, se reconoce que no hay una jerarquía entre curadores por ser mujer u hombre. Es claro para ellos que el poder de sanar viene de un espíritu que escogió a una persona para vivir en ella y darle el don de prevenir, diagnosticar y tratar una enfermedad causada por seres del mundo-otro. Es decir, dentro del pueblo wayuu no se decide ser una outsü por creer tener el don o por querer prepararse para serlo: a la mujer o al hombre le llega un espíritu, este los escoge y da aviso por medio de una experiencia onírica o a través de una enfermedad que es tratada por otra outsü. En ese momento la persona decide si aceptarlo e iniciar el proceso de preparación para ser una curadora o curador wayuu o pedirle a una outsü que le quite el espíritu, para lo cual ese ser pide que paguen por haber rechazado el don. Ese pago puede estar representado en animales de colores específicos, collares y la realización de la danza ritual wayuu: la yonna.

En la literatura antropológica wayuu se ha abordado el tema de las y los curadores outsü y outshi de forma conjunta porque ambos tienen las mismas facultades de curación, ya que el proceso curativo es guiado por los aseyuu -espíritus auxiliares- que se comunican a través del cuerpo de la persona que escogieron. En ese sentido, no es posible en este pueblo transmitir los conocimientos sobre curaciones espirituales de una persona a otra de manera arbitraria o según algún criterio que beneficie a unos u otros. A pesar de que en muchas investigaciones sociales sobre chamanismo y temas relacionados con salud y enfermedad, curadores y otras formas de sanación, predominó por mucho tiempo la perspectiva androcéntrica, en el caso wayuu no se encuentra claramente definido este sesgo porque las 
investigaciones abordan a hombres y mujeres curadores en conjunto y los trabajos son hechos igualmente por investigadores e investigadoras tanto wayuu como no wayuu; pero esto sí ocasionó en algunos casos el que se omitiera que la práctica curativa ritual es ejercida mayoritariamente por mujeres y que son ellas a las que consideran en su pueblo con la facultad de cuidar a su familia y su comunidad, las que dirigen los rituales en el que participan mujeres y hombres, y son las que se encargan de llevar a cabo lo que se revela en los sueños.

\section{Conclusiones}

En conclusión, en este trabajo analicé la práctica curativa wayuu en contextos rituales que lleva a cabo la mujer curadora -outsü- como autoridad espiritual en la comunidad de Wararalain y cómo se mantienen activas estas prácticas y saberes en el siglo XXI, teniendo en cuenta el contexto político, ambiental, cultural y de salud del que hace parte la realidad histórica que ha vivido el pueblo wayuu en Colombia. Esto reveló que, dentro de la comunidad, a pesar de contar con un servicio médico gratuito por ser indígenas, atienden sus enfermedades primero en el sistema curativo wayuu que es el que determina el tipo de enfermedad que padece una persona. Si es provocada por un wanülüü o yoluja, seres espirituales, el proceso curativo continúa con la outsü; de no tener una enfermedad de ese tipo, acuden al sistema biomédico para ser atendidos en un centro de salud público o tratan la enfermedad en sus casas con plantas medicinales.

La investigación pudo establecer que los mundos en los que transita la outsü, este mundo y el mundo-otro, constituyen un espacio de saber y diálogo donde su conocimiento y la práctica en rituales de sanación se definen por el carácter comunitario de las relaciones intersubjetivas entre las personas y los seres espirituales que intervienen en ritual de curación. Esas relaciones y diálogos son posibles, por un lado, por la conexión que hace la outsü con su espíritu auxiliar por medio del sonido de la maraca, el zumo del tabaco, el chirrinchi y su voz en el momento de la práctica curativa y, por el otro, a través de las experiencias oníricas de la outsü, del enfermo y un familiar de este, en donde los espíritus del mundo-otro usan el sueño como un espacio para comunicar lo que acontecerá y dar indicaciones específicas de cómo proceder en casos de enfermedad.

Todos esos aspectos caracterizan parte del sistema curativo wayuu y dentro de la comunidad de Wararalain son complementados, en casos específicos, con la asistencia al sistema biomédico con el cual se genera una articulación desde la comunidad, pero también evidencia tensiones desde el tratamiento biomédico, ya que desconocen el universo espiritual y simbólico que configura la práctica curativa ritual wayuu que es opuesta al carácter científico de la biomedicina. A pesar del desconocimiento sobre las formas en que la comunidad wayuu cura algunas enfermedades y de las relaciones y redes familiares de solidaridad que se activan en momentos de padecimientos de alguna enfermedad, en los centros públicos de salud, para acercarse a un modelo intercultural de salud pública, implementan una atención diferencial: los wayuu de Wararalain mencionan que existe la traductora, la trabajadora social, la prioridad en la atención por llegar de lugares alejados en las zonas rurales, la existencia de casas de paso para aquellos wayuu que no puedan regresar a tiempo a sus comunidades y hasta las brigadas de salud cada dos o tres meses con servicios de odontología, medicina general y un ginecólogo. Este último ya no llega a esa comunidad porque las mujeres no usaban ese servicio. En la comunidad wayuu de Wararalain consideran que ese tipo de 
atención está bien, pero todavía hay vacíos de los que deben ocuparse y quedan como recomendación a futuras investigaciones, puesto que las dificultades en la garantía del derecho a la salud es una problemática estructural que no se ha resuelto en décadas y todavía aqueja a las comunidades.

\section{Agradecimientos}

Agradezco a la comunidad de Wararalain por recibirme y compartir conmigo sus experiencias e historias; a cada una de las personas con las que conversé y que me permitieron recorrer su territorio, habitar sus espacios, participar de aspectos de sus vidas durante los tres meses de campo; a Michael Uzendoski por leer y corregirme cada documento que le envié para que fuera posible este artículo. A la Facultad Latinoamericana de Ciencias Sociales, FLACSO sede Ecuador, por el apoyo financiero a la investigación a través de las becas de tesis.

\section{Referencias}

Almendro, M. (2008). Chamanismo: La vía de la mente nativa. Barcelona: Kairós, S.A.

Araujo, D., y Finol, J. (2010). Sueño y sintaxis ritual entre los wayuu: análisis de la ceremonia de asülajawaa. Revista de Artes y Humanidades UNICA, 11(1), 71-106.

Balza-García, R. (2010). El mal, jerarquía y función socio-simbólica en la cultura Wayüu. Un enfoque simbólico. Espacio Abierto Cuaderno Venezolano de Sociología, 19(1), 93-115.

Bernard, R. (1995). Métodos de investigación en Antropología: abordajes cuantitativos y cualitativos. Estados Unidos de América: Altamira Press.

Bilhaut, A. (2011). El sueño de los záparas: Patrimonio onírico de un pueblo de la Alta Amazonía. Quito, Ecuador: Abya-Yala/Flacso, sede Ecuador.

Bidou, P., y Michel, P. (1988). Lenguaje y palabras chamánicas. Quito, Ecuador: AbyaYala.

Castro, D. (2017). La expansión del VIH y Sida: percepciones y respuestas sociales en los Wayuu de Colombia (tesis doctoral). Universidad de Granada, España.

Colpron, A. (2005). Monopólio masculino do xamanismo amazônico: O contra-exemplo das mulheres xamã shipibo-conibo. Mana 11(1), 95-128.

Comisión Interamericana de Derechos Humanos. (2015). Resolución 60/2015. Medidas cautelares No. 51-15. Niñas, niños y adolescentes de las comunidades de Uribia, Manaure, Riohacha y Maicao del Pueblo Wayúu, asentados en el departamento de La Guajira, respecto de Colombia.

Departamento Administrativo Nacional de Estadísticas. (2005). Censo general 2005. Recueprado de https://www.dane.gov.co/index.php/estadisticas-portema/demografia-y-poblacion/censo-general-2005-1

Departamento Administrativo Nacional de Estadísticas. (2018). Pobreza Monetaria y Multidimensional en Colombia 2017. Boletín técnico: pobreza monetaria La Guajira. Recuperado de DANE Información estratégica. http://www.dane.gov.co/files/investigaciones/condiciones_vida/pobreza/2017/Guaji ra_Pobreza_2017.pdf

Departamento Administrativo Nacional de Estadísticas. (2019). Población Indígena de Colombia: Resultados del Censo Nacional de población y vivienda 2018. Recuperado 
de

https://www.dane.gov.co/files/investigaciones/boletines/gruposetnicos/presentacion-grupos-etnicos-2019.pdf

Dejusticia. (2018). Informe sobre la situación de los derechos de las comunidades indígenas de La Guajira. Recuperado de https://cdn.dejusticia.org/wpcontent/uploads/2018/09/Informe-Guajira-2018.pdf

Eliade, M. (2001). El chamanismo y las técnicas arcaicas del éxtasis. México: Fondo de Cultura Económica.

Evans-Pritchard, E. (1976). Brujería, magia y oráculo entre los azande. Barcelona: Anagrama.

Harner, M. (2016). La senda del chaman. Barcelona: Kairos, S.A.

Ingold, T. (2004). Culture On The Ground. The World Perceived Through The Feet. Journal of Material Culture 9(3), 315-40. DOI: https://doi.org/10.1177/1359183504046896

Ingold, T. (2015). Conociendo desde dentro: Reconfigurando las relaciones entre antropología y etnografía. Etnografías Contemporáneas, 2(2), 218-232.

Instituto Nacional de Estadística. (2011). Resultados Población Indígena: XIV censo de población y vivienda 2011. Gerencia general de estadísticas demográficas. Instituto Nacional de Estadística. Recuperado de http://www.ine.gov.ve/documentos/Demografia/CensodePoblacionyVivienda/pdf/R esultadosBasicos.pdf

IPES Elkartea, Instituto, Promoción Estudios Sociales, Red de mujeres indígenas Seymaka, Fuerza de mujeres Wayuu y Wayunkerra Indigenous Women's Initiative. (2015). Desde el desierto: Sobre pueblos indígenas y una catástrofe sistemáticamente inducida. El caso del pueblo Wayuи en Colombia. Informe presentado ante el Comité para la eliminación de la discriminación racial, Ginebra, periodo de sesiones 87. Recuperado de https://tbinternet.ohchr.org/Treaties/CERD/Shared\%20Documents/COL/INT_CER D_NGO_COL_21216_S.pdf

Jaramillo, A. (2018). Sueños que guían, enseñan y recuerdan los sueños en la cultura Wayuu (tesis de pregrado). Pontificia Universidad Javeriana, Bogotá, Colombia.

Langdon, E. (1996). ¿Mueren en realidad los chamanes?: narraciones de los siona sobre chamanes muertos. Alteridades 6(12), 61-75.

Langdon, E. (2014). La negociación de lo oculto: Chamanismo, medicina y familia entre los Siona del bajo Putumayo. Popayán: Universidad del Cauca.

León, L. (2010). Chamanismo ancestral indígena en el encuentro del sí mismo. Bogotá: Universidad Cooperativa de Colombia (Educc).

Lévi-Strauss, C. (1995). Antropología estructural. Barcelona: Paidos, S.A.

Morillo, A., y Paz, C. (2008). Los sueños y su importancia en el pronóstico y tratamiento de la vivienda de los wayuu en Venezuela. Gazeta de Antropología, 24(2), 1-15. http://hdl.handle.net/10481/6960

Narby, J., y Huxley, F. (Ed.). (2005). Chamanes a través de los tiempos: Quinientos años en la senda del conocimiento. Barcelona: Kairos, S.A.

Ojeda, G. (2013). Contexto social y lingüístico de la mujer Ouutsü. Autoridad espiritual Wayuu. Colombia: Verdessa.

Paz, C., Leal, M., Alarcón, J., Valbuena, C., García, N., y Flores D. (2010). Ser curador Wayuu en la globalización y no morir en el intento. Index de Enfermería 19(2-3), 8892. 
Paz, C. (2007). Sistema explicativo del proceso salud-enfermedad en niños wayuu. Propuestas para la promoción de salud desde la interculturalidad. Maracaibo: Ediciones del Vice Rectorado Académico, Universidad del Zulia.

Paz, C. (2017). Hacer los sueños. Una perspectiva wayuu. Entre Diversidades, 277-288.

Perrin, M. (1987). Shamanistic Symptoms or Symbols? A Case of Indetermination: (The Body of the GuajiroShaman). Anthropos, 567-580.

Perrin, M. (1988). Formas de comunicación chamánica: el ejemplo guajiro (Venezuela y Colombia). En P. Bidou y M. Perrin (Coord.), Lenguaje y palabrras chamánicas (pp. 61-79). Quito: Abya-Yala.

Perrin, M. (1992). Los Practicantes del sueño: el chamanismo Wayüu. Caracas: Monte Ávila.

Perrin, M. (2011). Cura chamánica, sueños y eficacia terapéutica: el ejemplo de los Wayuu. En G. Freire. (Ed.), Perspectivas en salud indígena: cosmovisión, enfermedad y políticas públicas (pp. 227-249). Quito, Ecuador: Abya-Yala.

Perruchon, M. (1997). Llegar a ser una mujer-hombre: chamanismo y relaciones de género entre los Shuar. En M. Perrin y M. Perruchon (Coord.), Complementariedad entre hombre y mujer: Relaciones de género desde la perspectiva amerindia (pp. 47-75). Quito, Ecuador: Abya-Yala.

Quiroz, E. (2015). La mujer y la medicina tradicional en el resguardo de Panán: El rostro detrás de la historia (tesis de pregrado). Universidad de Nariño, San Juan de Pasto.

Rincón, L. (2006). Enfermarse y curarse en La Guajira: la salud entre tradición y modernidad. $\begin{array}{llll}\text { Revista } & \text { PRAXIS } & \text { 19-33. }\end{array}$ http://www.revistas.una.ac.cr/index.php/praxis/article/view/4651/4478

Serrano, S. (2020). Curaciones Wayuu en contextos rituales: la práctica de la outsü en La Guajira, Colombia (Tesis de Maestría). Facultad Latinoamericana de Ciencias Sociales - Flacso Ecuador, Quito, Ecuador.

Sherzer, J. (1988). El arte verbal de los cantos shamanísticos Cuna. En P. Bidou y M. Perrin (Coord.), Lengujae y palabras chamánicas (pp. 49-60). Quito, Ecuador: Abya-Yala / MLAL (Movimiento Laicos para América Latina).

Taussig, M. (2002). Chamanismo, colonialismo y el hombre salvaje: Un estudio sobre el terror y la curación. Bogotá D.C.: Norma.

Tedlock, B. (2005). The Woman in the Shaman's Body: Reclaiming the Femenine in Religion and Medicine. Nueva York: Bantam Books.

Tuhiwai-Smith, L. (2016). A descolonizar las metodologías: Investigación y pueblos indigenas. Santiago de Chile: LOM ediciones.

Turner, V. (1999). La selva de los símbolos. Aspectos del ritual ndembu. Madrid: Siglo XXI. Vasco, L. (2002). Entre selva y páramo: Viviendo y pensando la lucha india. Bogotá: ICANH. 